\title{
Jasmonate-dependent plant defense restricts thrips performance and preference
}

\author{
Hiroshi Abe*1, Takeshi Shimoda ${ }^{2}$, Jun Ohnishi ${ }^{3}$, Soichi Kugimiya ${ }^{4}$, \\ Mari Narusaka ${ }^{5}$, Shigemi Seo ${ }^{6}$, Yoshihiro Narusaka ${ }^{5}$, Shinya Tsuda ${ }^{2}$ and \\ Masatomo Kobayashi ${ }^{1}$
}

Address: ${ }^{1}$ Experimental Plant Division, RIKEN BioResource Center, Tsukuba 305-0074, Japan, ${ }^{2}$ National Agricultural Research Center, Tsukuba 305-8666, Japan, ${ }^{3}$ National Institute of Vegetable and Tea Science, Tsu 514-2392, Japan, ${ }^{4}$ National Institute for Agro-Environmental Sciences, Tsukuba 305-8604, Japan, ${ }^{5}$ Research Institute for Biological Sciences, Okayama 716-1241, Japan and ${ }^{6}$ National Institute of Agrobiological Sciences, Tsukuba 305-8666, Japan

Email: Hiroshi Abe* - ahiroshi@rtc.riken.jp; Takeshi Shimoda - oligota@affrc.go.jp; Jun Ohnishi - jonishi@affrc.go.jp; Soichi Kugimiya - kugimiya@affrc.go.jp; Mari Narusaka - ma_narusaka@bio-ribs.com; Shigemi Seo - sseo71@affrc.go.jp; Yoshihiro Narusaka - yo_narusaka@bio-ribs.com; Shinya Tsuda - shinyat@affrc.go.jp; Masatomo Kobayashi - kobayasi@rtc.riken.jp

* Corresponding author

Published: 27 July 2009

BMC Plant Biology 2009, 9:97 doi:10.1 I86/147I-2229-9-97
Received: 23 January 2009

Accepted: 27 July 2009

This article is available from: http://www.biomedcentral.com/I47I-2229/9/97

(c) 2009 Abe et al; licensee BioMed Central Ltd.

This is an Open Access article distributed under the terms of the Creative Commons Attribution License (http://creativecommons.org/licenses/by/2.0), which permits unrestricted use, distribution, and reproduction in any medium, provided the original work is properly cited.

\begin{abstract}
Background: The western flower thrips (Frankliniella occidentalis [Pergande]) is one of the most important insect herbivores of cultivated plants. However, no pesticide provides complete control of this species, and insecticide resistance has emerged around the world. We previously reported the important role of jasmonate (JA) in the plant's immediate response to thrips feeding by using an Arabidopsis leaf disc system. In this study, as the first step toward practical use of JA in thrips control, we analyzed the effect of JA-regulated Arabidopsis defense at the whole plant level on thrips behavior and life cycle at the population level over an extended period. We also studied the effectiveness of JA-regulated plant defense on thrips damage in Chinese cabbage (Brassica rapa subsp. pekinensis).
\end{abstract}

Results: Thrips oviposited more on Arabidopsis JA-insensitive coil-I mutants than on WT plants, and the population density of the following thrips generation increased on coil-I mutants. Moreover, thrips preferred coil-I mutants more than WT plants. Application of JA to WT plants before thrips attack decreased the thrips population. To analyze these important functions of JA in a brassica crop plant, we analyzed the expression of marker genes for JA response in $B$. rapa. Thrips feeding induced expression of these marker genes and significantly increased the JA content in $B$. rapa. Application of JA to $B$. rapa enhanced plant resistance to thrips, restricted oviposition, and reduced the population density of the following generation.

Conclusion: Our results indicate that the JA-regulated plant defense restricts thrips performance and preference, and plays an important role in the resistance of Arabidopsis and B. rapa to thrips damage. 


\section{Background}

Insect attack is one of the most important factors retarding plant growth, decreasing crop productivity, and causing other agricultural problems. A constitutive and inducible plant defense response confers immunity to herbivorous insects [1-3]. Analyses at the molecular, metabolic, and physiological levels $[2,4]$ have focused on responses to lepidopteran larvae (caterpillars) and aphids. Many analyses of plant responses to feeding by caterpillars have been conducted [e.g., [5-7]]. Caterpillars harm plants by chewing-type feeding, the best understood of several feeding modes. Although caterpillar feeding and mechanical wounding are physically similar, plants show obvious specific responses to caterpillar feeding [8]. Some of these responses are induced by insect gut and oviposition $[9,10]$. The sucking-type feeding by aphids and whiteflies is also well understood. However, in contrast to caterpillar feeding, sucking-type feeding rarely causes mechanical damage to the host plant. Rossi et al. [11] reported that the nematode resistance $(R)$ gene Mi-1 of tomato is involved in resistance to the potato aphid. Mi-1 also confers resistance to whiteflies [12]. Other major classes of insect feeding are also known. Leafminers feed within leaves and stems, forming tunnels (mining-type feeding), and thrips and spider mites feed by piercing and sucking $[13,14]$.

The western flower thrips (Frankliniella occidentalis [Pergande]) is one of the most important insect herbivores. This tiny insect tends to occupy narrow crevices within or between plant parts. The emergence worldwide of insecticide resistance among western flower thrips makes them difficult to control [15]. The thrips can also act as a vector of tospoviruses such as tomato spotted wilt virus $[16,17]$. Damage by western flower thrips is increasing in many countries; in particular, injury in greenhouse production is serious [18-20]. Thus, the development of new methods to control thrips damage by using the molecular mechanisms of plant responses is needed.

Jasmonate (JA) has an important function in plant responses to caterpillars and aphids [2]. Reymond et al. [21] reported that the JA-insensitive coi1-1 mutant of Arabidopsis is less resistant to cabbage butterfly (Pieris rapae). Ellis et al. [22] reported that coi1-1 mutants are less resistant to aphids, but the constitutive JA-signaling mutant cev1 is more resistant. Our recent study focusing on Arabidopsis response to thrips feeding also indicated the important function of JA $[23,24]$, and comparative transcriptome analyses suggested a strong relationship between JA treatment and thrips feeding [23]. Several groups reported that JA-regulated gene expression is induced by spider mites feeding $[25,26]$, which have a similar feeding mode to that of thrips. De Vos et al., using Arabidopsis genome arrays [27], also reported the impor- tance of JA for feeding-inducible gene expression by thrips and cabbage butterfly attack. Interestingly, they indicated the existence of common genes in the response to both feeding modes, and genes specific to each feeding mode.

Arabidopsis is a widely studied experimental plant for which many useful genomic resources and much other information are available. However, it is not suitable for analyzing Arabidopsis responses to caterpillars, which can quickly eat an entire plant. On the other hand, with the tiny western flower thrips, it is possible to analyze Arabidopsis responses to thrips attack over generations.

In this study, we focused on the effect of JA-regulated Arabidopsis defense at the whole plant level on thrips behavior and life cycle at the population level. We analyzed the long-term effects of JA-regulated plant defense on thrips oviposition, the population density of the following thrips generation (larvae and pupae), and preference between Arabidopsis WT and JA-insensitive coi1-1 mutant host plants. The results show important effects of the JAdependent plant defense on both thrips performance and preference. In addition, application of JA to Arabidopsis WT plants before thrips attack decreased the thrips population. Expression analyses of marker genes for JA response in Chinese cabbage (Brassica rapa subsp. pekinensis) suggested the occurrence of a JA-dependent defense against thrips attack in this plant, too. The JA content of $B$. rapa was significantly increased after thrips feeding, and application of JA to plants enhanced their resistance to thrips.

\section{Results \\ Importance of jasmonate-regulated Arabidopsis defense in resistance to thrips attack}

We recently reported the role of JA in the short-term response of Arabidopsis to thrips feeding on leaf discs over 1 or 2 days $[23,24]$. To analyze its role in long-term defense at the whole plant level, we compared the feeding damage between whole WT plants and JA-insensitive coil1 mutants [28] inoculated with 20 thrips at 3 weeks. The coi1-1 mutants had been completely devoured by 4 weeks after inoculation, whereas WT plants were flowering and producing siliques (Fig. 1A). These results suggest the importance of JA-regulated defense in the resistance of Arabidopsis to thrips attack.

To understand why coi1-1 mutants showed low resistance to thrips attack, we first analyzed the number of thrips eggs on the WT plants and coi1-1 mutants to compare the asexual oviposition performance of thrips. Arabidopsis rosette leaves were cut into leaf discs with 8-mm diameter. One adult female thrips was put on each disc and allowed to feed and oviposit. Because the females lay in the epidermal or mesophyll cells [29], we stained the eggs with 


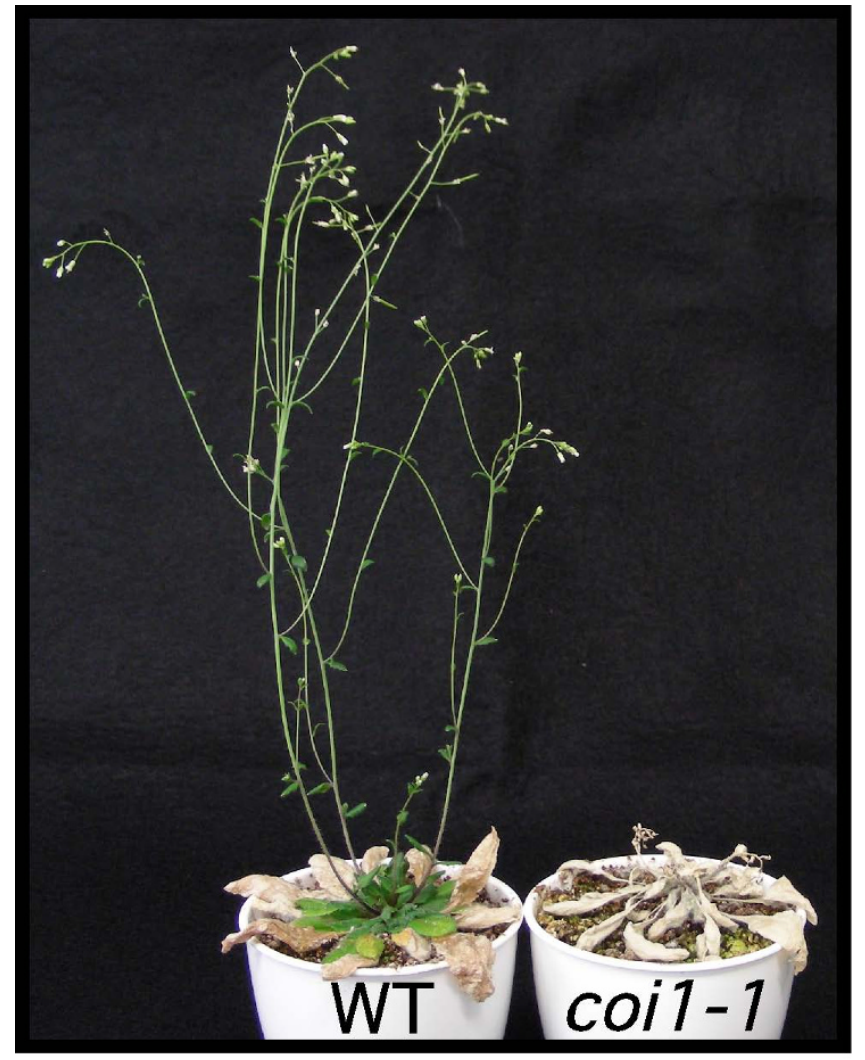

Figure I

Function of JA in plant resistance to thrips feeding. Twenty adult females fed on 3-week-old WT plants (left) or coil-I mutants (right). Typical plants after 4 weeks of feeding are shown.

trypan blue to count them. As we reported previously [23], the area of feeding scars on coil-1 mutants was greater than that on WT plants (data not shown). The number of eggs on the coil-1 discs was double that on the WT discs (Fig. 2A-C). The decreased resistance of these coi1-1 mutants could explain the increased oviposition rate on these plants.

\section{Effect of jasmonate-dependent Arabidopsis defense on thrips population}

Because the JA-regulated defense affected oviposition, we analyzed its effect on the subsequent generation. We put 20 adult females on WT and coi1-1 plants and counted adults, larvae, and pupae after 2 weeks. We covered the soil with fine zirconia beads $0.4 \mathrm{~mm}$ in diameter to find thrips easily. Thrips fed much more on coi1-1 mutants than on WT plants (Fig. 3A, B). About 14 of the original adult females remained on coi1-1 mutants, but only about 2 remained on WT plants (Fig. 3C). Similarly, while more than 190 larvae lived on the coi1-1 mutants, only about 20 lived on the WT plants (Fig. 3D). We also found 5 times as many pupae on coi1-1 mutants than on WT plants (Fig.
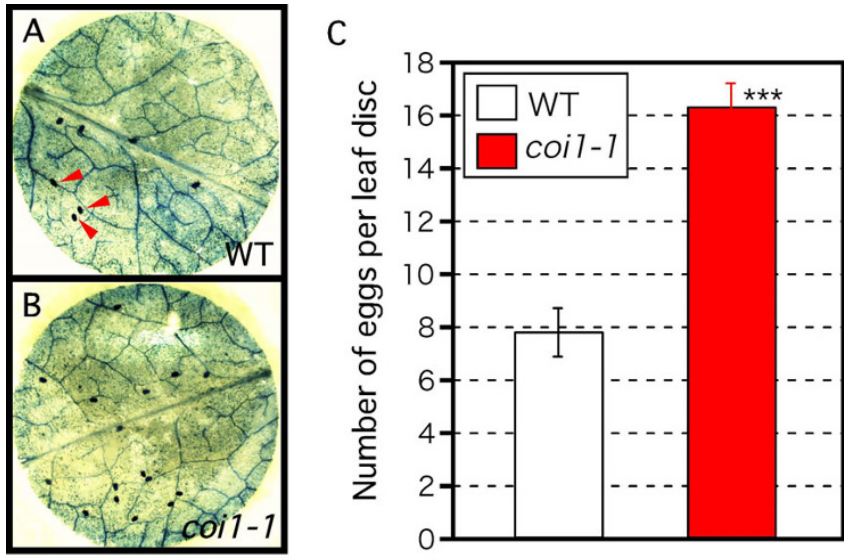

\section{Figure 2}

Effect of JA-dependent plant resistance on thrips oviposition on leaf discs. One adult female fed per leaf disc of 3-week-old WT plants (A) or coil-I mutants (B) for 4 days. Eggs oviposited on leaf discs were stained with trypan blue. Photos show typical leaf discs after staining; some eggs are shown by red arrowheads. (C) Number of eggs per leaf disc (mean \pm SD) based on more than 10 independent determinations. Asterisks indicate significant difference (Student's $t$ test), ${ }^{* * *} \mathrm{p}<0.00 \mathrm{I}$.

3E). These results demonstrate that the JA-regulated defense can determine thrips population size.

Next, we analyzed the effect of JA-regulated plant defense on host plant preference of thrips. We placed 100 adult females halfway between WT and coi1-1 plants (Fig. 4A) and counted the thrips on each plant after 2 days. The coi1-1 mutants had many more thrips than the WT plants (Fig. $4 \mathrm{~A}):>70 \%$ versus about $5 \%\left(\chi^{2}\right.$ test, $\chi^{2}=175.879, \mathrm{df}$ $=1, \mathrm{p}<0.001$; Fig. $4 \mathrm{~B})$; the remaining thrips roamed the surroundings. These results indicate that the JA-regulated plant defense influences the host plant preference of thrips.

We next analyzed the effect of JA treatment on Arabidopsis resistance to thrips attack. JA-treated plants had half as many eggs as untreated plants (Fig. 5A). The numbers of adults and larvae showed a similar contrast (Fig. 5B, C). Together with the results from the coil-1 mutants, these results indicate that the JA-dependent defense response in Arabidopsis plays an important role in resisting thrips.

Jasmonate-dependent plant resistance to thrips in $\mathrm{B}$. rapa To search for JA-dependent resistance to thrips in a brassica crop, we analyzed the function of JA in B. rapa, one of the most important brassica crops in the world, especially in Asia. A search of the B. rapa EST database (National Center for Biotechnology Information) revealed putative counterparts of Arabidopsis JA-inducible 
A $\quad$ B

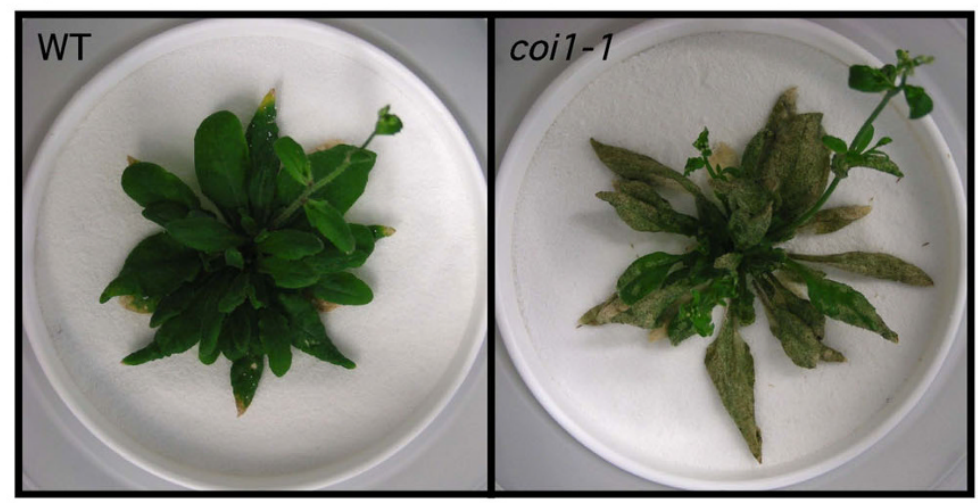

C

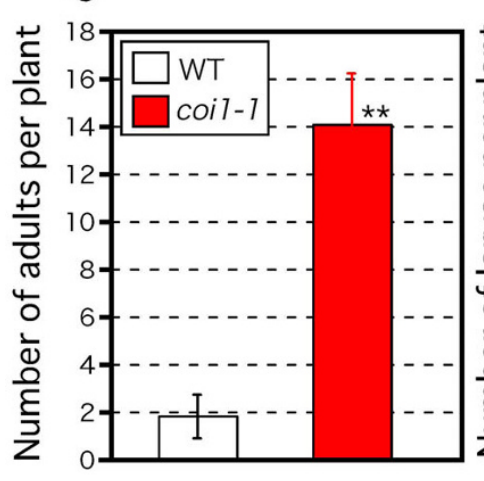

D

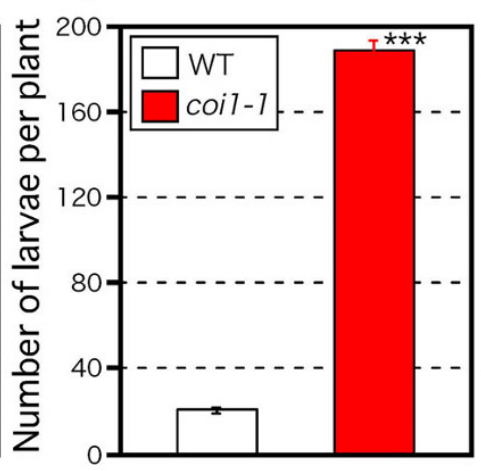

$\mathrm{E}$

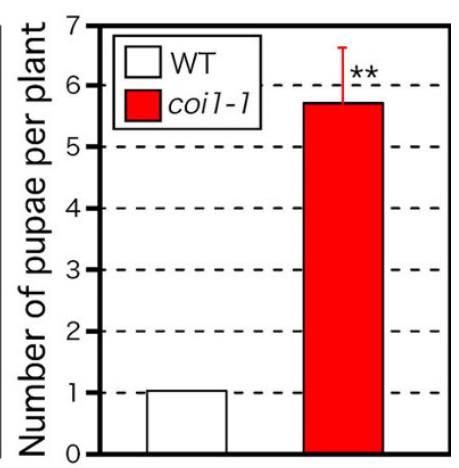

\section{Figure 3}

Effect of the JA-dependent plant defense on thrips population. (A, B) Twenty adult females fed on 3-week-old WT plants (A) or coil-I mutants (B) for 2 weeks. (C-E) Number of adults (C), larvae (D), and pupae (E) after 2 weeks; mean \pm SD based on five independent determinations. Asterisks indicate significant differences (Student's $t$-test), ${ }^{* *} p<0.005,{ }^{* * *} p<0.00 \mathrm{I}$.

marker genes. We analyzed the expression of marker genes of the JA pathway corresponding to AtVSP2 and AtLOX2, and genes corresponding to allene oxide synthase (AtAOS) and allene oxide cyclase 2 (AtAOC2), both of which encode enzymes that catalyze JA biosynthesis as shown by previous reports (Fig. 6E) [30,31]. Expression of the brassica counterparts, BrVSP2, BrLOX2, BrAOS, and BrAOC2, was induced by thrips feeding (Fig. 6A-D). In addition, the JA content of $B$. rapa plants infested by thrips was significantly higher than that of control plants (one-way ANOVA, $F=13.938$, df = 2, $\mathrm{p}<0.01$; Fig. $6 \mathrm{~F})$. These data suggest the involvement of JA in the response to thrips feeding in B. rapa also.

To confirm the functional role of JA in plant resistance to thrips attack in B. rapa, we analyzed the effect of JA treatment on thrips feeding. Injury from thrips attack was lower in plants treated with JA than in untreated plants (Fig. 7A-D), by a factor of about 15 (Fig. 7E). These results indicate that the JA-dependent plant defense against thrips is conserved in B. rapa.
We further analyzed JA's effect on thrips oviposition. Rosette leaves of B. rapa were cut into leaf discs with 8-mm diameter. One adult female thrips was put on each leaf disc and allowed to feed and oviposit for 4 days. Application of JA dose-dependently decreased the number of eggs (one-way ANOVA, $F=10.367$, df = 4, p < 0.001; Fig. 8A). Finally, we analyzed the effect of JA on the next generation. JA treatment of plants restrained the thrips population very effectively (Fig. 8B, C). These results clearly indicate the important role of JA in resistance to thrips attack in B. rapa also.

\section{Discussion}

The phytohormone JA regulates part of a plant's basal defense system. Numerous studies have examined the functions of JA in plant responses to pathogen attack, mechanical wounding, UV irradiation, ozone exposure, osmotic stress [32,33], and insect feeding [34,35]. The JAZ (jasmonate ZIM-domain) family of repressors was identified in Arabidopsis as a negative regulator of JA signaling [36-38]. JAZ interacts with COI1 protein, degrades, and so 
A

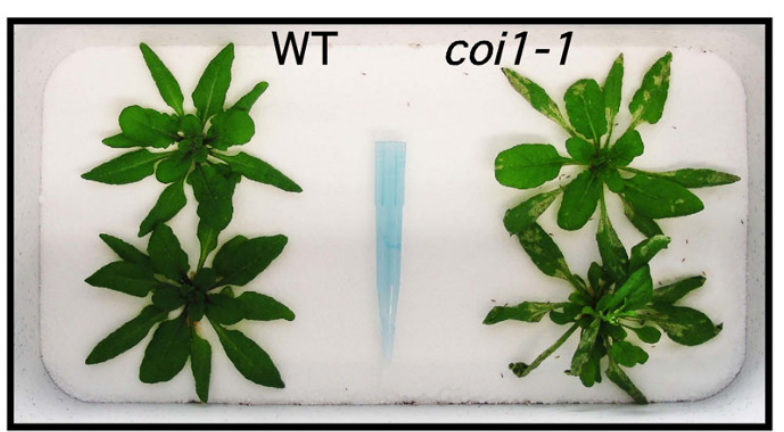

B

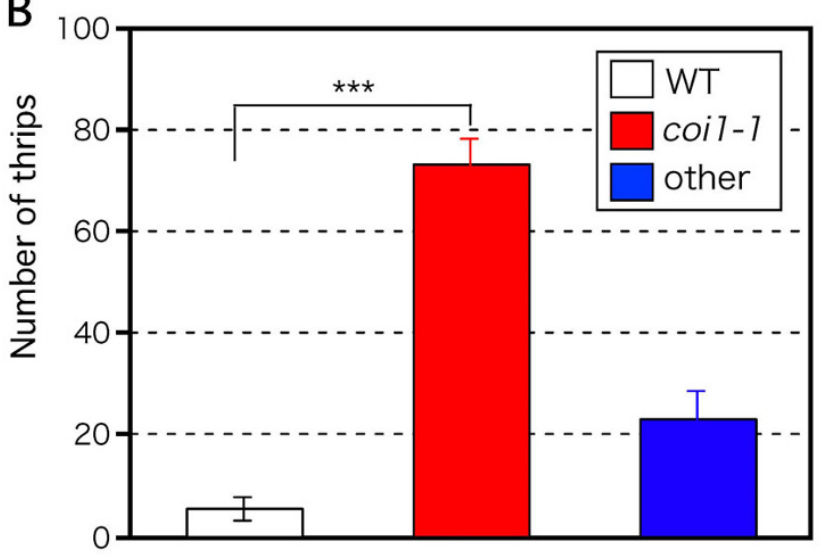

Figure 4

Effect of the JA-dependent plant defense on host plant preference of thrips. (A) Three-week-old WT plants (left) and coil-I mutants (right) were grown at each end of a pot. One hundred adult females were collected in a $\mathrm{I}-\mathrm{mL}$ tube and laid between the plants. Photo shows plants after 2 days. (B) Number of adult thrips on each plant after 2 days. Mean \pm SD based on five independent determinations. Asterisk indicates a significant difference between the two plants, $\chi^{2}$ test, ${ }^{* * *} p<0.001$. The remaining thrips roamed the surroundings were excluded from the statistical analysis.

induces JA-responsive gene expression. Overexpression of a modified form of JAZ1 significantly decreased plant resistance to the beet armyworm (Spodoptera exigua) [39]. Resistance by coi1-1 mutants to cabbage butterfly caterpillar (Pieris rapae) was similarly decreased [21].

However, these analyses focused on plant responses to lepidopteran larvae. Because caterpillars quickly devour Arabidopsis plants and change to butterflies or moths, which fly away, it is difficult to analyze the Arabidopsis response and insect performance over generations on the one Arabidopsis plant. For these reasons, we used thrips. We found differences in symptoms between WT plants and JA-insensitive coi1-1 mutants: thrips had demolished coi1-1 mutants after 4 weeks, yet WT plants had flowers and siliques (Fig. 1A). As it seemed unlikely that only 20 adult thrips could kill a plant in 4 weeks, we also studied the effect of a JA-dependent Arabidopsis defense on oviposition. The number of eggs on coil-1 was about double that on the WT (Fig. 2A-C). As we described previously [23], the area of feeding scars in coil-1 was much greater than that on WT plants (data not shown). The greater number of eggs on coi1-1 might result from the better performance of adult thrips. Alternatively, a difference in plant metabolites between WT and coil-1 might influence oviposition. Annadana et al. [40] reported that cysteine protease inhibitors restrict oviposition by western flower thrips. Wounding and JA induce many genes encoding cysteine protease inhibitors [41], including Arabidopsis cystatin-1 (AtCYS1) [42]. Cysteine protease inhibitors could explain the difference in thrips oviposition between WT and coi1-1 plants.

Next, we analyzed the effect of JA-regulated plant defense on the population density of the following generation of thrips. Surprisingly, the population increased around 10fold after 2 weeks on the coi1-1 mutants, but changed little on the WT plants (Fig. 3A-E). Most of the thrips on coi1-1 were larvae. We found some dead larvae on the WT plants but none on coil-1 (data not shown). These results indicate that the JA-dependent plant defense in WT plants reduces the survival of thrips larvae. We found about 7 times as many adult thrips on coil-1 as on the WT, which indicates that thrips can survive longer on coi1-1. We attribute the much greater population of thrips on coil-1 to this increased longevity and the greater egg production on coi1-1 mutants, and the higher mortality of larvae on the WT plants. Analysis of the hatching rate of eggs could also help explain the increased population on coi1-1. Barth et al. [43] reported that a double knock-out mutant of Arabidopsis lacking two major genes for myrosinase (tgg1, tgg2), which degrades glucosinolates to toxins such as isothiocyanates, showed decreased resistance to the cabbage looper (Trichoplusia ni) and tobacco hornworm (Manduca sexta). Sasaki-Sekimoto et al. [33] reported that JA regulates glucosinolate biosynthesis. Recently, Shroff et al. [44] showed that the preferential allocation of glucosinolates to the periphery of leaves may play a key role in the defense of leaves by creating a barrier to chewing herbivores, which frequently approach leaves from the edge. Several other compounds protect plants against insect pests. Konno et al. [45] reported that cysteine proteases such as papain, ficin, and bromelain showed toxicity to two notorious pests, cabbage armyworm (Mamestra brassicae) and cotton leafworm (Spodoptera litura). They later reported that sugar-mimic alkaloids were toxic to cabbage armyworm [46]. Further analyses will help to explain which kinds of compounds, regulated by JA, reduce thrips performance.

The choice test showed that coil-1 mutants attracted 14 times as many thrips as did WT plants (Fig. 4A, B). As a result, coi1-1 mutants suffered more damage. Aharoni et 
A

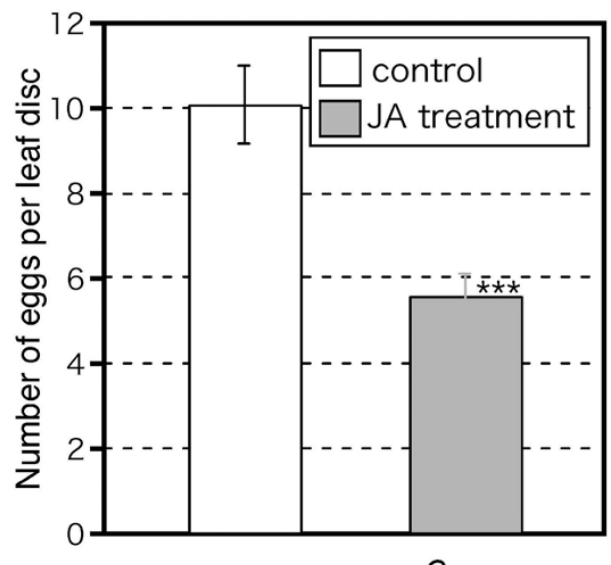

B

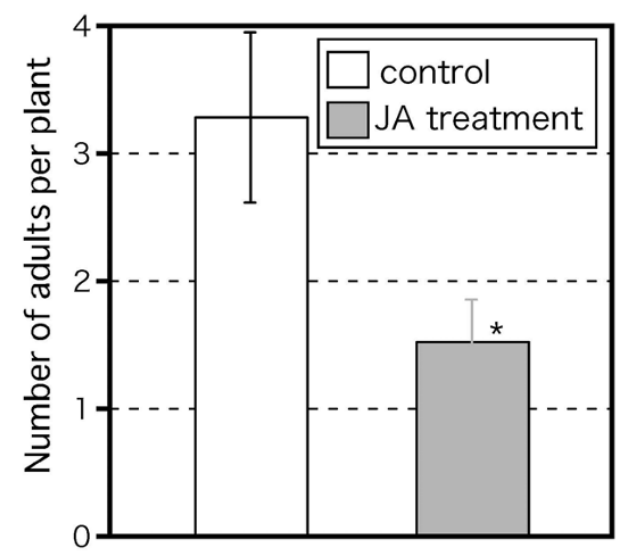

C

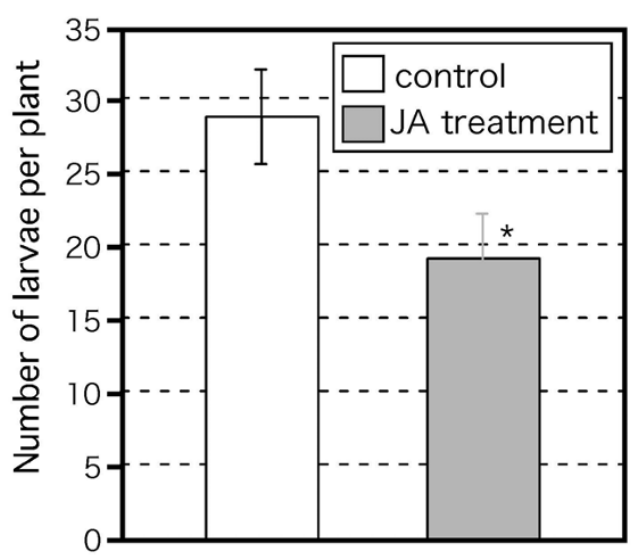

\section{Figure 5}

Effect of JA-induced Arabidopsis defense response on thrips population. Twenty adult females fed on 3-week-old WT plants. Either $50 \mu \mathrm{M}$ JA or water (control) was applied 2 days before thrips were introduced. After 2 weeks, eggs (A), adults (B), and larvae (C) were counted. Mean \pm SD based on five independent determinations. Asterisks indicate significant differences (Student's t-test), ${ }_{p}<0.05, *^{*} *_{p}<0.00$ I.

al. [47] reported that overexpression of a gene for a dual linalool/nerolidol synthase (FaNES1) in Arabidopsis, which produces those two terpenes, enhances avoidance by green peach aphids (Myzus persicae). Interestingly, these FaNES1-overexpressing plants also attracted carnivorous predatory mites (Phytoseiulus persimilis) [48]. JAdeficient spr2 tomato plants emit less herbivory-induced volatiles and attract more tobacco hornworm and tobacco whitefly (Bemisia tabaci) for oviposition [49]. In addition to the volatile components, many other plant metabolites such as nutrient factors and toxic compounds are reported as stimulants or deterrents of host plant preference [50]. These metabolic components may explain the higher preference of the thrips for coil-1 mutants or higher avoidance of WT plants.

The western flower thrips is one of the most serious insect herbivores in the world. It is also a virus vector. Because of its thigmokinetic behavior and the emergence of insecticide resistance, it is difficult to control with insecticides [15]. Therefore, new control methods are urgently needed. Application of JA to WT Arabidopsis plants before thrips damage decreased the thrips population (Fig. 5AC). We previously reported that thrips feeding induced in Arabidopsis expression of AtVSP2 and AtLOX2 (marker genes of the JA pathway) and AtAOS1 and AtAOC2 (encoding allene oxide synthase and allene oxide cyclase), which catalyze JA biosynthesis in Arabidopsis [23]. Here, the expression of their counterparts in $B$. rapa was also induced by thrips feeding (Fig. 6A-D), as was the JA content (Fig. 6F), as reported previously in Arabidopsis [23]. These results indicate that the JA-dependent defense system is conserved between Arabidopsis and B. rapa. Interestingly, JA application also greatly decreased the amount of feeding scars in B. rapa (Fig. 7A-E), and decreased egg production and thrips population size (Fig. 8A-C). The effect 
A

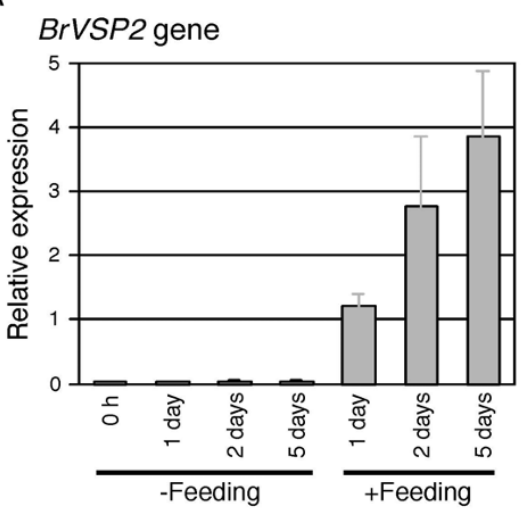

C

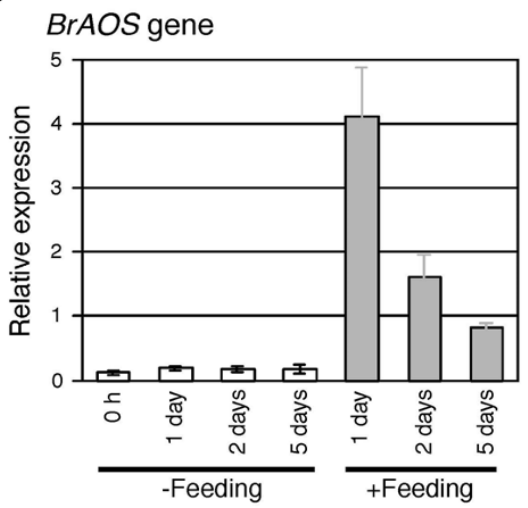

E

Linolenic Acid (LA)<smiles></smiles>

12-oxo-phytodieonic acid (OPDA)

$\downarrow$
$\downarrow$
$\downarrow$
$\downarrow$
†
OPR3
B

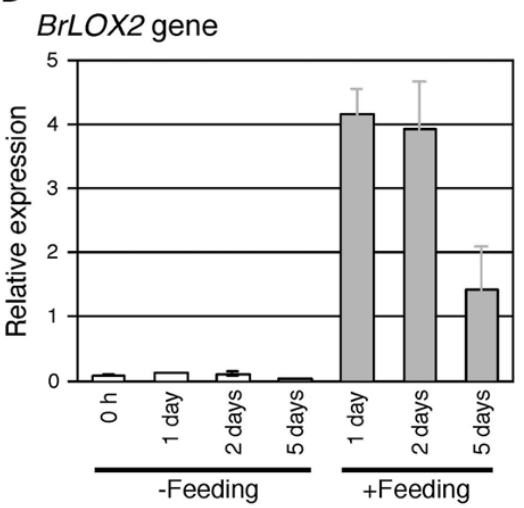

D

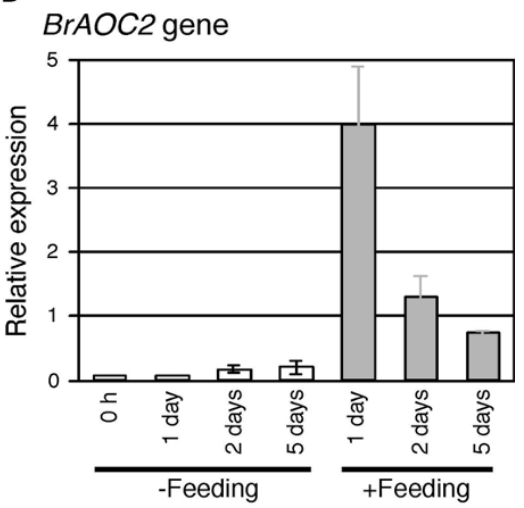

$\mathrm{F}$

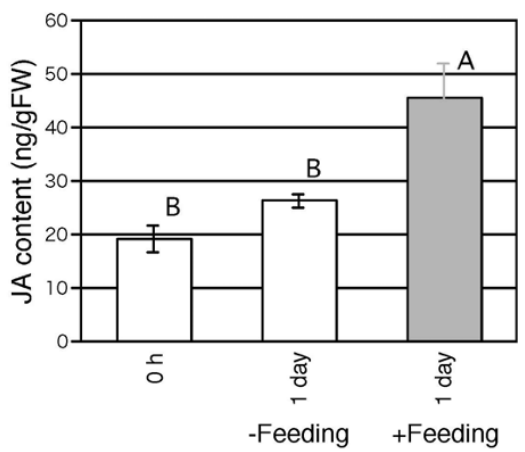

\section{Figure 6}

Involvement of JA signaling in B. rapa response to thrips feeding. (A-D) Expression of marker genes for JA response in B. rapa was induced by thrips feeding. BrVSP2 (A), BrLOX2 (B), BrAOS (C), and BrAOC2 (D) are brassica counterparts of Arabidopsis marker genes of the JA pathway and JA biosynthesis. Twenty-five adult females fed on five 2-week-old plants per pot. After 0 , I, 2, and 5 days, total RNA was prepared from the plants with (+Feeding) or without (-Feeding) thrips, and first-strand cDNA was synthesized for PCR analysis. The expression level of each gene was normalized to the expression of BrACT2 (control). Mean \pm SD based on three replications. (E) Proposed model of the biosynthesis of JA in Arabidopsis. (F) Effect of thrips feeding on the biosynthesis of JA in B. rapa. Ten adult females fed on a 2-week-old plant (+Feeding). A control plant was kept without thrips (-Feeding). At the beginning of the experiment $(0 \mathrm{~h})$ and after I day from the start of feeding, I $g$ of plant tissue was sampled for measurement of endogenous JA (JA + methyl JA). Means \pm SD of three independent measurements. Different letters indicate statistically significant differences between treatments (Tukey-Kramer HSD test; $p<0.05$ ). 


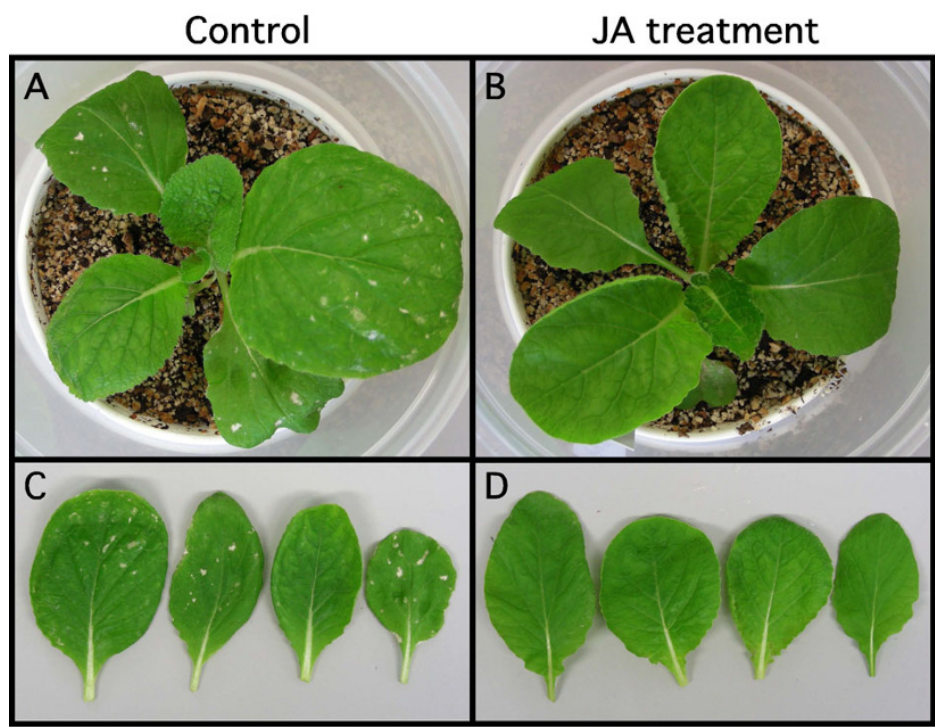

$\mathrm{E}$

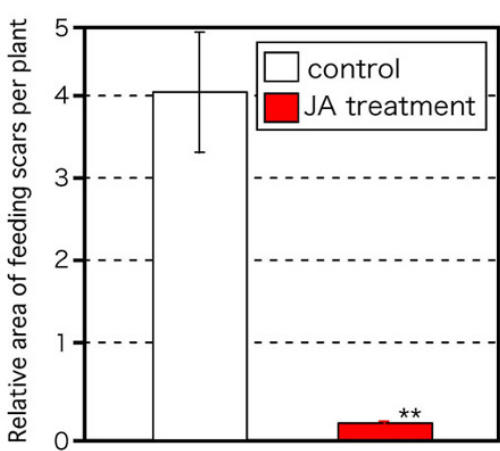

\section{Figure 7}

Effect of JA application on plant resistance to thrips. Twenty adult females fed on 2-week-old B. rapa plants for 10 days. Water (control; A, C) or $50 \mu \mathrm{M}$ JA (B, D) was applied I day before thrips were introduced. (E) Mean \pm SD of area of feeding scars based on more than 10 independent determinations. Asterisk indicates significant difference (Student's $t$-test), $* * p<$ 0.005 .

of JA application was much higher in B. rapa than in Arabidopsis, but the biological significance of this difference is unclear. Several groups have combined JA-mediated transcriptome analyses with metabolomics data [33,51]. Further comparative analyses between B. rapa and Arabidopsis using these approaches are needed to explain the differences in plant resistance. The genome of $B$. rapa is being sequenced http://brassica.bbsrc.ac.uk/. In the near future, Brassica 'omics' analyses using genome information will be available. Comparative expression analyses between $B$. rapa and Arabidopsis suggested the existence of similar and specific responses to pathogen infection in these species [52].

Jasmonate application to Nicotiana sylvestris plants decreased plant biomass [53]. Overexpression of AtJMT in Arabidopsis plants, which leads to elevated JA level [54], decreased the flower number and total seed weight significantly. Importantly, Thaler et al. showed that although application of JA in tomato fields successively decreased naturally occurring thrips, spray application at low concentration $(0.5 \mathrm{mM})$ decreased neither plant biomass nor fruit production [55]. However, the effect of low JA concentration on thrips control is lower than that of high JA concentration $(1.5 \mathrm{mM})$. JA application incurs costs for plant fitness, and also activates plant defense, which must be balanced for optimum production. The screening of the specific compounds to regulate plant defense to insect attack will be a promising approach.

\section{Conclusion}

In this study, as the first step toward practical use of JA in thrips control, we analyzed the effect of JA-regulated Arabidopsis defense at the whole plant level on thrips behavior and life cycle at the population level. Our results indicate that JA-regulated Arabidopsis defense restricts both thrips performance and preference. Thrips performance was evaluated from oviposition and the population density of 
A

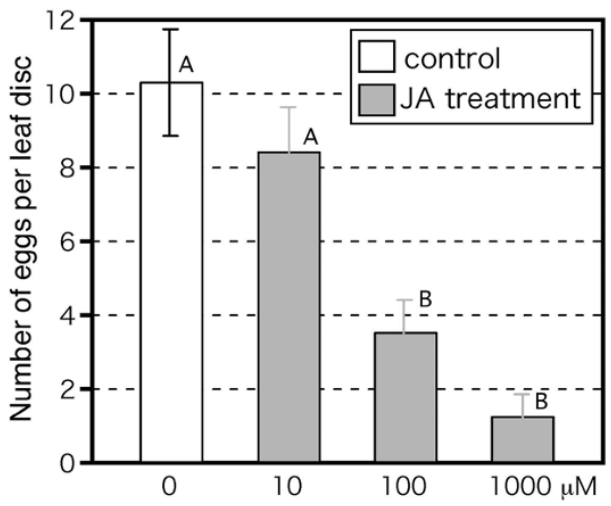

B

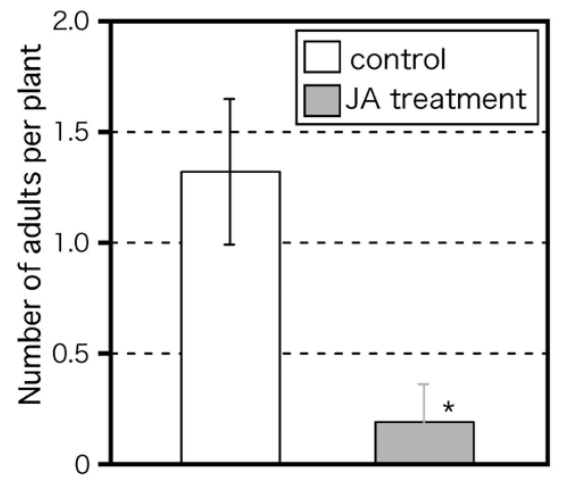

C

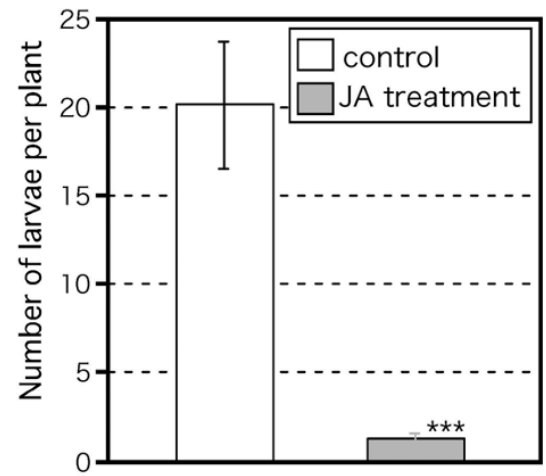

Figure 8

Effect of JA-induced B. rapa defense response on thrips population. (A) Water (control) or 10, 100 , or $1000 \mu \mathrm{M} J \mathrm{~A}$ was applied to 2-week-old B. rapa plants I day before thrips were introduced. One adult female fed on each leaf disc for 4 days. Eggs were stained with trypan blue. Mean \pm SD of eggs per leaf disc based on 10 independent determinations. Different letters indicate statistically significant differences between treatments (Tukey-Kramer HSD test; $p<0.05$ ). (B, C) Twenty adult females fed on 2-week-old WT plants for 2 weeks. Water (control) or $50 \mu \mathrm{M}$ JA was applied I day before thrips were introduced. After 2 weeks, adults $(B)$ and larvae $(C)$ were counted. Mean \pm SD based on five independent determinations. Asterisks indicate significant differences (Student's $t$-test), ${ }^{*} p<0.05, *^{* *} p<0.00$ I.

the following generation. The effect of JA-regulated defense on thrips population density was considerable. This was due to the effects on thrips longevity, egg production, and mortality of larvae. Fully understanding the plant defense against thrips attack will require determination of the actual plant metabolites that restrict thrips performance and preference.

In B. rapa also, induction of expression of marker genes for the JA pathway and increased JA content after thrips damage support the occurrence of a JA-dependent defense against thrips attack. JA application to $B$. rapa greatly decreased feeding damage on account of decreased egg production and thrips population density. The existence of diverse targets of JA-regulated plant defense indicates that JA concurrently regulates multiple responses involved in plant resistance to thrips damage. JA-regulated plant defense could be a good target for practical applications to control thrips.

\section{Methods}

\section{Plant materials and cultivation}

Wild-type (ecotype Col-0) Arabidopsis plants and the JA signaling and biosynthesis mutant coil-1 were grown in soil as described previously [56]. Briefly, seeds were sown on sterile soil in pots, moistened, and held at $4^{\circ} \mathrm{C}$ for 7 days in the dark to synchronize germination. The pots were then transferred to $22^{\circ} \mathrm{C}$ with a long-day photoperiod (16 $\mathrm{h}$ light $/ 8 \mathrm{~h}$ dark). Plants at the four-leaf stage were transferred individually to pots and grown to the rosette stage. Chinese cabbage (B. rapa subsp. pekinensis cv. Kyoto No. 3, Takii Seed Co. Ltd., Kyoto, Japan) plants were grown similarly. 


\section{Identification of coil - I plants}

Homozygous coil-1 plants were selected according to PCR amplification of a sequence of the Arabidopsis COI1 gene followed by digestion with BsmI (TOYOBO, Osaka, Japan). Within the amplified PCR product, the BsmI restriction site is present only in the coi1-1 mutant. Primers were as follows: forward, 5'-GGAAACAGGAGCCCGAGATC-3'; reverse, 5'-TGGATGTTTCTCGGAGCAGC-3'.

\section{Thrips attack}

Laboratory colonies of Frankliniella occidentalis were maintained in a closed environmental chamber, as described previously [57]. The assay used female adults 14-21 days after emergence from the pupal stage. The adults were starved for 2 to $3 \mathrm{~h}$ before feeding on test plants. Twenty adult females were allowed to feed on each whole plant in a cylindrical acryl chamber with air ventilation windows covered with a fine mesh.

\section{Jasmonate treatment}

Pots holding 3-week-old Arabidopsis plants or 2-week-old B. rapa plants grown in soil were transferred into a cylindrical acryl chamber containing $100 \mu \mathrm{M}$ JA solution. Other experiments to count the number of eggs on $B$. rapa leaf discs used 10, 100, or $1000 \mu \mathrm{M}$ JA solution. JA treatment was carried out for 2 days before the beginning of thrips attack.

\section{Counting of thrips eggs}

Leaf discs with 8-mm diameter were cut with a biopsy punch (Kay Industries, Oyana, Japan). The discs were floated on $1.5 \mathrm{~mL}$ of distilled water in wells of a white 1.5mL sample tube stand (Assist, Tokyo, Japan). A single adult female that had been starved for 2 to $3 \mathrm{~h}$ was placed on each leaf disc. The sample tube stand was covered with ABI Prism Optical Adhesive Cover (Applied Biosystems, Foster City, CA, USA), and a few tiny holes for air were made with a 27-G fine injection needle. Thrips were allowed to feed and oviposit for 4 days at $22^{\circ} \mathrm{C}$. Eggs were stained with trypan blue as described previously [58].

\section{Counting of the thrips population}

Three-week-old Arabidopsis plants or 10-day-old B. rapa plants grown in soil covered with fine zirconia beads (Nikkato Co., Osaka, Japan; $0.4 \mathrm{~mm}$ in diameter to make it easy to find the thrips) were placed in a cylindrical acryl chamber as above. Twenty adult females were put on each plant. After 2 weeks, the adults, larvae, and pupae were counted.

\section{Choice assay}

Three-week-old WT and coi1-1 plants grown in soil covered with fine zirconia beads in a white pot $(255 \times 145 \times$ $120 \mathrm{~mm}$; Appleware, Osaka, Japan) were used for a choice assay in a cylindrical acryl chamber as above. Each pot held four plants (two of each type) separated by $150 \mathrm{~mm}$. One hundred adult females were deposited halfway between the plants and allowed to move freely. After 2 days, the thrips on each plant were counted.

\section{Quantitative reverse transcription PCR}

Twenty-five female adult thrips fed on five 2-week-old $B$. rapa plants at the rosette stage for 1, 2, or 5 days in a closed container with air vents. Experiments were repeated twice. After feeding, the plants were frozen in liquid nitrogen. Total RNA $(2 \mu \mathrm{g})$ isolated with Trizol reagent (Invitrogen, Carlsbad, CA, USA) and an RNeasy MinElute Cleanup Kit (Qiagen, Valencia, CA, USA) was treated with RNase-free DNase (Takara) to eliminate genomic DNA. First-strand cDNA was synthesized with random oligo-hexamers and Superscript III reverse transcriptase according to the manufacturer's instructions (Invitrogen). Quantitative realtime PCR was carried out with Power SYBR Green PCR Master Mix (Applied Biosystems) using the first-strand cDNA as a template on a sequence detector (ABI Prism $7900 \mathrm{HT}$, Applied Biosystems). Expression of BrACT2 was used for normalization. Nucleotide sequences of the genespecific primers were as follows: BrVSP2 (forward, 5'GACTCCAAAACGGTGTGCAAA-3'; reverse, 5'AGGGTCTCGTCAAGGTCAAAGA-3'); BrLOX2 (5'-TCCC CACTTCCGCTACACC-3'; 5'-AATACTTTCCGGGCCAGA AAC-3'); BrAOS (5'-GATCTCCCCATCCGAACCAT-3'; 5'AACTCCTCGGGTTTTTGCTTG-3'); BrAOC2 (5'-GCCGGTCTCTGTGTCTTGATC-3'; 5'-ACGGACAGGTGGCCATAGTC-3'); and BrACT2 (5'-ACCCAAAGGCCAACAGAG AG-3'; 5'-CTGGCGTAAAGGGAGAGAACA-3').

\section{Jasmonate quantification}

JA and its methyl ester were quantified as described previously [47], except that an HP6890 gas chromatograph fitted to a quadrupole mass spectrometer (Hewlett-Packard, Wilmington, DE, USA) was used. Approximately $1 \mathrm{~g}$ of each $B$. rapa plant with or without thrips feeding was used for quantification. Three independent samples were analyzed.

\section{Measurement of the area of feeding scars}

The area of thrips feeding scars on the surface of each $B$. rapa leaf was measured using WinROOF software, version 5.8.1 (Mitani Corporation, Tokyo, Japan), on digitized images taken under a VHX-200 digital microscope (Keyence, Osaka, Japan).

\section{Statistics}

The results of thrips oviposition, population density and feeding activity were respectively subjected to Student's $t$ test or analysis of variance (one-way ANOVA) followed by Tukey-Kramer HSD test. The result from choice assay was subjected to a $\chi^{2}$ test; the null hypothesis was that thrips exhibited a 50:50 distribution over WT and coi1-1 plants. 
These analyses were performed the JMP software, ver. 5.1 (SAS Institute, Inc., Cary, NC, USA).

\section{Accession numbers}

The GenBank accession numbers for the genes mentioned in this article are as follows: BrVSP2 (EX101964), BrLOX2 (EX100417), BrACT2 (EX137335), BrAOS (EX104579), BrAOC2 (EX125486).

\section{Authors' contributions}

HA planned the study and carried out the feeding assay, thrips performance and preference analyses, and wrote the manuscript. TS and SK performed thrips performance and preference analyses and participated in final writing of the manuscript. JO did the measurement of the area of feeding scars. MN and $\mathrm{YN}$ did the gene expression analyses of Brassica. SS performed JA quantification. ST and MK planned the study and participated in its coordination and final writing of the manuscript. All authors read and approved the final manuscript.

\section{Acknowledgements}

We thank F. Mori, S. Kawamura, and I. Sasaki of RIKEN BRC and S. Nagai and Y. Matsumura of the National Agricultural Research Center for their excellent technical assistance. We also thank Dr. M. Watanabe of NIAS for his kind support and helpful discussion. This work was supported by a Grant-in-Aid for Scientific Research for a "Young Scientist (B)" from the Ministry of Education, Culture, Sports, Science and Technology to HA, and by a grant from the 2004 Industrial Technology Research Grant Program of the New Energy and Industrial Technology Development Organization of Japan to $\mathrm{YN}$ and $\mathrm{HA}$.

\section{References}

I. Kessler A, Baldwin IT: Plant responses to insect herbivory: the emerging molecular analysis. Annu Rev Plant Biol 2002, 53:299-328.

2. Howe GA, Jander G: Plant immunity to insect herbivores. Annu Rev Plant Biol 2008, 59:4I-66.

3. Howe GA, Schaller A: Direct defenses in plants and their induction by wounding and insect herbivores. In Induced Plant Resist ance to Herbivory Edited by: Schaller A. New York, Springer; 2008:7-29.

4. Van Poecke RMP: Arabidopsis-insect interactions. The Arabidopsis Book 2008 [http://www.aspb.org/publications/arabidopsis/]. Rockville, MD: American Society of Plant Biologists

5. Kahl J, Siemens DH, Aerts RJ, Gabler R, Kuhnemann F, Preston CA, Baldwin IT: Herbivore-induced ethylene suppresses a direct defense but not a putative indirect defense against an adapted herbivore. Planta 2000, 21 0:336-342.

6. Winz RA, Baldwin IT: Molecular interactions between the specialist herbivore Manduca sexta (Lepidoptera, Sphingidae) and its natural host Nicotiana attenuata. IV. Insect-induced ethylene reduces jasmonate-induced nicotine accumulation by regulating putrescine $\mathbf{N}$-methyltransferase transcripts. Plant Physiol 200 I, I 25:2 189-2202.

7. Li C, Liu G, Xu C, Lee GI, Bauer P, Ling HQ, Ganal MW, Howe GA The tomato suppressor of prosystemin-mediated responses2 gene encodes a fatty acid desaturase required for the biosynthesis of jasmonic acid and the production of a systemic wound signal for defense gene expression. Plant Cell 2003, I 5:|646-1661.

8. Reymond P, Weber H, Damond M, Farmer EE: Differential gene expression in response to mechanical wounding and insect feeding in Arabidopsis. Plant Cell 2000, 12:707-7I9.
9. Korth KL: Profiling the response of plants to herbivorous insects. Genome Biol 2003, 4:22I.I-22I.4.

10. Little D, Gouthier-Darimont C, Bressow F, Reymond P: Oviposition by pierid butterflies triggers defense responses in Arabidopsis. Plant Physiol 2007, 143:784-800.

II. Rossi M, Goggin FL, Milligan SB, Kaloshian I, Ullman DE, Williamson VM: The nematode resistance gene $M i$ of tomato confers resistance against the potato aphid. Proc Natl Acad Sci 1998, 95:9750-9754.

12. Nombela G, Williamson VM, Muniz M: The root-knot nematode resistance gene Mi-l.2 of tomato is responsible for resistance against the whitefly Bemisia tabaci. Mol Plant Microbe Interact 2003, 16:645-649.

13. Geest LPS Van Der: Aspect of physiology. In Spider mite: their biology, natural biology, natural enemies and control Edited by: Helle W, Sabelis MW. Amsterdam, Elsevier; 1985: I7I-184.

14. Parrella MP: IPM: approaches and prospects. In Thrips Biology and Management Edited by: Parker BL, Skinner M, Lewis T. New York, Plenum; 1995:357-364.

15. Jensen SE: Insecticide resistance in the western flower thrips, Frankliniella occidentalis. Integr Pest Manag Rev 2000, 5: I3 I- I 46.

16. Wijkamp I, van Lent J, Kormelink R, Goldbach R, Peter D: Multiplication of tomato spotted wilt virus in western flower thrips. In Thrips Biology and Management Edited by: Parker BL, Skinner M, Lewis T. New York, Plenum; 1995:357-364.

17. Whitfield AE, Ullman DE, German TL: Tospovirus-thrips interactions. Annu Rev Phytopathol 2005, 43:459-489.

18. Lewis T: Pest thrips in perspective. In Thrips as Crop Pests Edited by: Lewis T. Cambridge, Cambridge University Press; 1997:I-I4.

19. Van Driesche Lyon S, Stanek EJ III, Xu B, Nunn C: Evaluation of efficacy of Neoseiulus cucumeris for control of western flower thrips in spring bedding crops. Biological Control 2006, 36:203-2I5.

20. Reitz SR: Biology and Ecology of the Western Flower Thrips (Thysanoptera: Thripidae): The Making of a Pest. Florida Entomologist 2009, 92:7-13.

21. Reymond P, Bodenhausen N, Van Poecke RM, Krishnamurthy V Dicke $M$, Farmer EE: A conserved transcript pattern in response to a specialist and a generalist herbivore. Plant Cell 2004, 16:3|32-3147.

22. Ellis C, Karafyllidis I, Turner JG: Constitutive activation of jasmonate signaling in an Arabidopsis mutant correlates with enhanced resistance to Erysiphe cichoracearum, Pseudomonas syringae, and Myzus persicae. Mol Plant Microbe Interact 2002, 15:1025-1030.

23. Abe H, Ohnishi J, Narusaka M, Seo S, Narusaka Y, Tsuda S, Kobayashi $M$ : Function of jasmonate in response and tolerance of Arabidopsis to thrips feeding. Plant Cell Physiol 2008, 49:68-80.

24. Abe H, Ohnishi J, Narusaka M, Seo S, Narusaka Y, Tsuda S, Kobayashi M: Arabidopsis-thrips system for analysis of plant response to insect feeding. Plant Signaling \& Behav 2008, 3: $1-2$.

25. Arimura G, Ozawa R, Shimoda T, Nishioka T, Boland W, Takabayashi J: Herbivory-induced volatiles elicit defence genes in lima bean leaves. Nature 2000, 406:5I2-5I5

26. Zheng SJ, van Dijk JP, Bruinsma M, Dicke M: Sensitivity and speed of induced defense of cabbage (Brassica oleracea L.): Dynamics of BoLOX expression patterns during insect and pathogen attack. Molecular Plant-Microbe Interactions 2007, 20: I332-I345.

27. De Vos M, Van Oosten VR, Van Poecke RMP, Van Pelt JA, Pozo MJ, Mueller MJ, Buchala AJ, Metraux JP, Van Loon LC, Dicke M, Pieterse CMJ: Signal signature and transcriptome changes of Arabidopsis during pathogen and insect attack. Molecular PlantMicrobe Interactions 2005, 18:923-937.

28. Xie DX, Feys BF, James S, Nieto-Rostro M, Turner JG: COII : an Arabidopsis gene required for jasmonate-regulated defense and fertility. Science 1998, 280:1091-1094.

29. Childers CC, Achor DS: Thrips feeding and oviposition injuries to economic plants, subsequent damage and host responses to infestation. In Thrips Biology and Management Edited by: Parker BL, Skinner M, Lewis T. New York, Plenum; 1995:3 I-5I.

30. Creelman RA, Mullet JE: Biosynthesis and action of jasmonates in plants. Annu Rev Plant Physiol Plant Molec Biol 1997, 48:355-38I.

31. Delker C, Stenzel I, Hause B, Miersch O, Feussner I, Wasternack C: Jasmonate biosynthesis in Arabidopsis thaliana : enzymes, products, regulation. Plant Biol (Stuttg.) 2006, 8:297-306. 
32. Thomma B, Eggermont K, Penninckx I, Mauch-Mani B, Vogelsang R, Cammue BPA, Broekaert WF: Separate jasmonate-dependent and salicylate-dependent defense-response pathways in Arabidopsis are essential for resistance to distinct microbial pathogens. Proc Natl Acad Sci USA 1998, 95:15107-15।III.

33. Sasaki-Sekimoto $Y$, Taki N, Obayashi T, Aono M, Matsumoto F, Sakurai N, Suzuki H, Hirai MY, Noji M, Saito K, Takamiya K, Shibata $\mathrm{D}$, Ohta $\mathrm{H}$ : Coordinated activation of metabolic pathways for antioxidants and defence compounds by jasmonates and their roles in stress tolerance in Arabidopsis. Plant J 2005, 44:653-668.

34. Schaller A, Stintzi A: Jasmonate biosynthesis and signaling for induced plant defense against herbivory. In Induced Plant Resistance to Herbivory Edited by: Schaller A. New York, Springer; 2008:349-366

35. Browse J, Howe GA: New weapons and a rapid response against insect attack. Plant Physiol 2008, 146:832-838.

36. Chini A, Fonseca S, Fernández G, Adie B, Chico JM, Lorenzo O, Garcia-Casodo G, Lopes-Vidriero I, Lozano FM, Ponce MR, Micol JL, Solano R: The JAZ family of repressors is the missing link in jasmonate signaling. Nature 2007, 448:666-67I.

37. Thines B, Katsir L, Melotto M, Niu Y, Mandaokar A, Liu G, Nomura $\mathrm{K}, \mathrm{He} \mathrm{SH}$, Howe GA, Browse J: JAZ repressor proteins are targets of the SCFCOII complex during jasmonate signaling. Nature 2007, 448:66I-665.

38. Yan Y, Stolz S, Chetelat A, Reymond P, Pagni M, Dubugnon L, Farmera $\mathrm{EE}$ : A downstream mediator in the growth repression limb of the jasmonate. Plant Cell 2007, I 5:2866-2884.

39. Chung HS, Koo AJK, Gao X, Jayanty S, Thines B, Jones AD, Howe GA Regulation and function of Arabidopsis JASMONATE ZIMdomain genes in response to wounding and herbivory. Plant Physiol 2008, I 46:952-964.

40. Annadana S, Peters J, Gruden K, Schipper A, Outchkourov NS, Beekwilder MJ, Udayakumar M, Jongsma MA: Effects of cysteine protease inhibitors on oviposition rate of the western flower thrips, Frankliniella occidentalis. J Insect Physiol 2002, 48:70I-706.

4I. Botella MA, Xu Y, Prebha TN, Zhao Y, Narasimhan ML, Wilson KA, Nielsen SS, Bressan RA, Hasegawa PM: Differential expression of soybean cysteine proteinase inhibitor genes during development and in response to wounding and methyl jasmonate. Plant Physiol 1996, I I 2: 1201-1210.

42. Belenghi B, Acconcia F, Trovato M, Perazzolli M, Bocedi A, Polticell F, Ascenzi P, Delledonne M: AtCYSI, a cystatin from Arabidopsis thaliana, suppresses hypersensitive cell death. Eur J Biochem 2003, 270:2593-2604

43. Barth C, Jander G: Arabidopsis myrosinases TGGI and TGG2 have redundant function in glucosinolate breakdown and insect defense. Plant J 2006, 46:549-562.

44. Shroff R, Vergara F, Muck A, Svatoš A, Gershenzon J: Nonuniform distribution of glucosinolates in Arabidopsis thaliana leaves has important consequences for plant defense. Proc Natl Acad Sci 2008, 105:6196-620I.

45. Konno K, Hirayama C, Nakamura M, Tateishi K, Tamura Y, Hattori M, Kohno K: Papain protects papaya trees from herbivorous insect: role of cysteine proteases in latex. Plant J 2004, 37:370-378.

46. Konno K, Ono H, Nakamura M, Tateishi K, Hirayama C, Tamura Y, Hattori M, Koyama A, Kohno K: Mulberry latex rich in antidiabetic sugar-mimic alkaloids forces dieting on caterpillars. Proc Natl Acad Sci 2006, 107: I337-I341.

47. Aharoni A, Giri AP, Deuerlein S, Griepink F, de Kogel WJ, Verstappen FW, Verhoeven HA, Jongsma MA, Schwab W, Bouwmeester H]: Terpenoid metabolism in wild-type and transgenic Arabidopsis plants. Plant Cell 2003, I5:2866-2884.

48. Kappers IF, Aharoni A, van Herpen TWJM, Luckerhoff LLP, Dicke M, Bouwmeester $\mathrm{HJ}$ : Genetic engineering of terpenoid metabolism attracts bodyguards to Arabidopsis. Science 200I, 23:2070-2072

49. Sánchez-Hernández C, López MG, Délano-Frier JP: Reduced levels of volatile emissions in jasmonate-deficient spr2 tomato mutants favour oviposition by insect herbivores. Plant Cell Environ 2006, 29:547-557.

50. Schoonhoven LM, van Loon JA, Dicke M: Host-plant selection: when to accept a plant. In Insect-plant biology Oxford, Oxford university press; 2005: 169-199.
5I. Pauwels L, Morreel K, De Witte E, Lammertyn F, Van Montagu M, Boerjan W, Inzé D, Goossens A: Mapping methyl jasmonatemediated transcriptional reprogramming of metabolism and cell cycle progression in cultured Arabidopsis cells. Proc Natl Acad Sci 2008, 105:। 380-1385.

52. Narusaka M, Abe H, Kobayashi M, Kubo Y, Narusaka Y: Comparative analysis of expression profiles of counterpart gene sets between Brassica rapa and Arabidopsis thaliana during fungal pathogen Colletotrichum higginsianum infection. Plant Biotechnol 2006, 23:503-508

53. Baldwin IT, Hamilton W: Jasmonate-Induced Responses of Nicotiana sylvestris Results in Fitness Costs Due to Impaired Competitive Ability for Nitrogen. Journal of Chemical Ecology 2000, 26:915-952.

54. Cipollini D: Consequences of the overproduction of methy jasmonate on seed production, tolerance to defoliation and competitive effect and response of Arabidopsis thaliana. New Phytologist 2006, I 73:146-153.

55. Seo HS, Song JT, Cheong J-J, Lee Y-H, Lee Y-W, Hwang I, Lee JS, Choi YD: Jasmonic acid carboxyl methyltransferase: A key enzyme for jasmonate-regulated plant responses. Proc Natl Acad Sci 200I, 95:9750-9754.

56. Thaler IS, Stout MJ, Karban R, Duffey SS: Jasmonate-mediated induced plant resistance affects a community of herbivores. Ecological Entomology 200I, 26:312-324.

57. Weigel D, Glazebrook J: How to grow Arabidopsis. In Arabidopsis: A Laboratory Manual Edited by: Weigel D, Glazebrook J. New York, Cold Spring Harbor Laboratory Press; 2002: I-I8.

58. Murai T, Loomans AJM: Evaluation of an improved method for mass-rearing of thrips and a thrips parasitoid. Entomol Exp Appl 200I, I 0I:28I-289.

59. Seo S, Okamoto M, Seto H, Ishizuka K, Sano H, Ohashi Y: Tobacco MAP kinase: a possible mediator in wound signal transduction pathways. Science 1995, 270:1988-1992.

Publish with BioMed Central and every scientist can read your work free of charge

"BioMed Central will be the most significant development for disseminating the results of biomedical research in our lifetime. "

Sir Paul Nurse, Cancer Research UK

Your research papers will be:

- available free of charge to the entire biomedical community

- peer reviewed and published immediately upon acceptance

- cited in PubMed and archived on PubMed Central

- yours - you keep the copyright
BioMedcentral 